\title{
Anomalous interaction of nonlocal solitons in media with competing nonlinearities
}

\author{
Esbensen, B. K.; Bache, Morten; Bang, Ole; Krolikowski, Wieslaw
}

Published in:

Physical Review A

Link to article, DOI:

10.1103/PhysRevA.86.033838

Publication date:

2012

Document Version

Publisher's PDF, also known as Version of record

Link back to DTU Orbit

Citation (APA):

Esbensen, B. K., Bache, M., Bang, O., \& Krolikowski, W. (2012). Anomalous interaction of nonlocal solitons in media with competing nonlinearities. Physical Review A, 86(3), 033838.

https://doi.org/10.1103/PhysRevA.86.033838

\section{General rights}

Copyright and moral rights for the publications made accessible in the public portal are retained by the authors and/or other copyright owners and it is a condition of accessing publications that users recognise and abide by the legal requirements associated with these rights.

- Users may download and print one copy of any publication from the public portal for the purpose of private study or research.

- You may not further distribute the material or use it for any profit-making activity or commercial gain

- You may freely distribute the URL identifying the publication in the public portal

If you believe that this document breaches copyright please contact us providing details, and we will remove access to the work immediately and investigate your claim. 


\title{
Anomalous interaction of nonlocal solitons in media with competing nonlinearities
}

\author{
B. K. Esbensen, ${ }^{1}$ M. Bache, ${ }^{1}$ O. Bang, ${ }^{1}$ and W. Krolikowski ${ }^{2}$ \\ ${ }^{1}$ DTU Fotonik, Department of Photonics Engineering, Technical University of Denmark, DK-2800 Kgs. Lyngby, Denmark \\ ${ }^{2}$ Laser Physics Centre, Research School of Physics and Engineering, Australian National University, Canberra, \\ Australian Capital Territory 0200, Australia
}

(Received 13 July 2012; published 25 September 2012)

\begin{abstract}
We theoretically investigate properties of individual bright spatial solitons and their interaction in nonlocal media with competing focusing and defocusing nonlinearities. We consider the general case with both nonlinear responses characterized by different strengths and degrees of nonlocality. We employ a variational approach to analytically describe soliton properties. In particular, we prove analytically that the interplay of focusing and defocusing nonlocal nonlinearities leads to attraction or repulsion of solitons depending on their separation distance. We then study the propagation and interaction of solitons using numerical simulations of the full model of beam propagation. The numerical simulations fully confirm our analytical results.
\end{abstract}

DOI: 10.1103/PhysRevA.86.033838

PACS number(s): 42.65.Tg, 03.75.Lm

\section{INTRODUCTION}

Solitons, i.e., nondiffracting localized wave structures, are formed thanks to an interplay between linear spreading (dispersion or diffraction), which tends to spread the wave and the self-induced nonlinear response (such as refractive index change) of the medium, which focuses the wave. In the stationary regime, these two processes are exactly in equilibrium, leading to wave self-trapping and its propagation as a soliton. Solitons are generic for a wide variety of nonlinear systems. They have been identified in nonlinear optical media, transmission lines, water waves, biological systems, cold matter, and many others. For instance, bright optical solitons propagate as finite-size self-trapped beams [1,2]. On the other hand, solitons in Bose-Einstein condensates represent coherent atomic structures formed due to competition between spreading caused by its kinetic energy and attracting interaction potential $[3,4]$.

The uniqueness of solitons lies in the fact that they are robust objects exhibiting particlelike properties in collisions $[1,2,5]$. In particular, solitons originating from integrable nonlinear models, collide elastically [6]. On the other hand, those governed by nonintegrable models, such as optical solitons in media with saturable nonlinearity, collide inelastically and may either experience fission or fusion upon collision [7]. Most of the studies of soliton interaction dealt with so-called local media where the nonlinearity in every point is determined solely by the wave intensity in that very point. However, it appears that in many nonlinear systems, the nonlinear response is spatially nonlocal. This means that the wave-induced nonlinear response in a particular location depends on the wave intensity in a certain neighborhood of this location [8]. In the case of highly nonlocal media, the nonlinear response is no longer a function of the beam intensity, but rather its total power [9]. The nonlocality of nonlinearity may be caused by either transport processes, such as heat conduction [10] or ballistic atomic transport [11] and diffusion [12], charge separation [13], or long-range particle interaction, as in dipolar Bose-Einstein condensates [14-16] or nematic liquid crystals [17-19]. It has also been demonstrated that a parametric nonlinear wave interaction, such as second-harmonic generation, is in fact well described by a nonlocal nonlinearity, which has enabled accurate descriptions of quadratic solitons [20], modulational instability [21], and soliton pulse compression [22-24] in quadratic nonlinear materials.

Apart from profoundly modifying properties of individual localized waves and solitons, e.g., collapse arrest of finite beams $[8,25,26]$ and stabilization of complex soliton structures such as the vortex [27-31], the nonlocal character of the nonlinearity has been shown to dramatically affect the interaction of solitons. In particular, nonlocality provides an attractive force between even remote solitons [9,32-36], leading to, e.g., attraction of otherwise repelling solitons and the formation of bound states of bright and dark solitons [37-42].

The nonlocal nonlinear response also appears in media with the so-called synthetic nonlinearities. Those are media where the nonlinearity results from two or more competing processes or effects, such as the cubic quintic response in generalized Kerr media [43,44]. A nonlocal competing nonlinear response occurs naturally in Bose-Einstein condensates with the simultaneous presence of local (contact) and nonlocal (dipole-dipole) nonlinear interaction potentials with their relative strengths and signs (repulsive or attractive) controlled by the experimental conditions [16,45-47].

Given the nonlocal nature of a parametric wave interaction [20], quadratic media, such as ferroelectric crystals, actually inherently constitute a system of competing nonlinearities as they simultaneously support local (Kerr) and nonlocal $\left(\chi^{(2)}\right)$ nonlinear responses. Early works have found, for example, that competition between those nonlinearities arrests collapse [48] and stabilizes solitons [49]. This has an important application in the compression of large-energy femtosecond pulses, since the dominating self-defocusing nonlinearity ensures that the input pulse energy can be scaled to arbitrarily large values, and therefore the energy limit of standard self-focusing compressors is removed [50,51].

Competing nonlinearities can also be realized in nematic liquid crystals where they involve both thermal and orientational nonlinear responses to the presence of a light beam [52]. The combination of fast local and slow nonlocal nonlinearities has been recently proposed as a way to create stable optical bullets [53]. Few recent works have analyzed the effect of competing nonlocal nonlinearities on the existence 
and stability of solitons. It has been shown recently that the simultaneous presence of nonlocal nonlinearities of opposite sign leads to stabilization of complex soliton structures, which are otherwise unstable in a medium with one type of nonlocal nonlinearity [54-56].

In their recent work, Du et al. have demonstrated numerically that competing nonlocal nonlinearities may drastically influence the character of soliton interaction. Namely, it appears that for a certain range of nonlinearity parameters, two in-phase bright solitons, which would normally attract, exhibit repulsion instead $[57,58]$.

In this work, we study the effect of competing nonlocal nonlinearity on the interaction of bright solitons. We employ a variational approach to demonstrate analytically that the competition of nonlocal focusing and defocusing with different spatial scales leads to anomalous repulsion of in-phase and incoherent bright solitons. We confirm our analytical results in numerical simulations.

\section{MODEL}

Here we will consider propagation of a one-dimensional beam with the slowly varying amplitude $u(x, z)$, where $x$ and $z$ are transverse and longitudinal coordinates, respectively. The evolution of this beam in media with the self-induced nonlinear refractive index change $\Delta n(x)$ is described by the following dimensionless nonlocal nonlinear Schrödinger (NLS) equation:

$$
i \frac{\partial u}{\partial z}+\frac{\partial^{2} u}{\partial x^{2}}+\Delta n u=0 .
$$

We will represent the nonlinear refractive index change of the medium using the following phenomenological model of competing focusing and defocusing nonlocal nonlinearities:

$$
\begin{aligned}
\Delta n(x)= & \alpha_{1} \Delta n_{1}(x, I)+\alpha_{2} \Delta n_{2}(x, I) \\
= & \alpha_{1} \int R_{1}\left(x-x^{\prime}\right) I\left(x^{\prime}, z\right) d x^{\prime} \\
& +\alpha_{2} \int R_{2}\left(x-x^{\prime}\right) I\left(x^{\prime}, z\right) d x^{\prime} .
\end{aligned}
$$

Here $\alpha_{1}$ and $\alpha_{2}$ represent the strength and the sign of the two nonlinear contributions, respectively, and $I=|u(x, z)|^{2}$ is the wave intensity. Here we will assume that $\alpha_{1}>0$ and $\alpha_{2}<0$, so they correspond to self-focusing and self-defocusing nonlinearities, respectively. The nonlocal response function $R_{1,2}(x)$ defines the nonlocal character of the nonlinearity. The actual form of this function is determined by the physics of the process responsible for the nonlocal response. In particular, $R_{i}(x)=\sigma_{i}^{-1} \exp \left(-|x| / \sigma_{i}\right)$ describes the nonlocal nonlinearity of nematic liquid crystals in one transverse dimension $[18,19]$. Here $\sigma_{i}(i=1,2)$ defines the width of the respective nonlocal response.

For the sake of analytical simplicity, in this work we will use a Gaussian nonlocal response function $R_{i}(x)=$ $1 / \sqrt{\pi \sigma_{i}} \exp \left(-|x|^{2} / \sigma_{i}^{2}\right)$. However, our results are applicable to other types of nonlocal response.

In typical media with a single nonlocal nonlinearity, the nonlocal response function is symmetric, exhibiting a single peak and decaying monotonically to zero. The sign of the function does not change with $x$. However, in the presence of
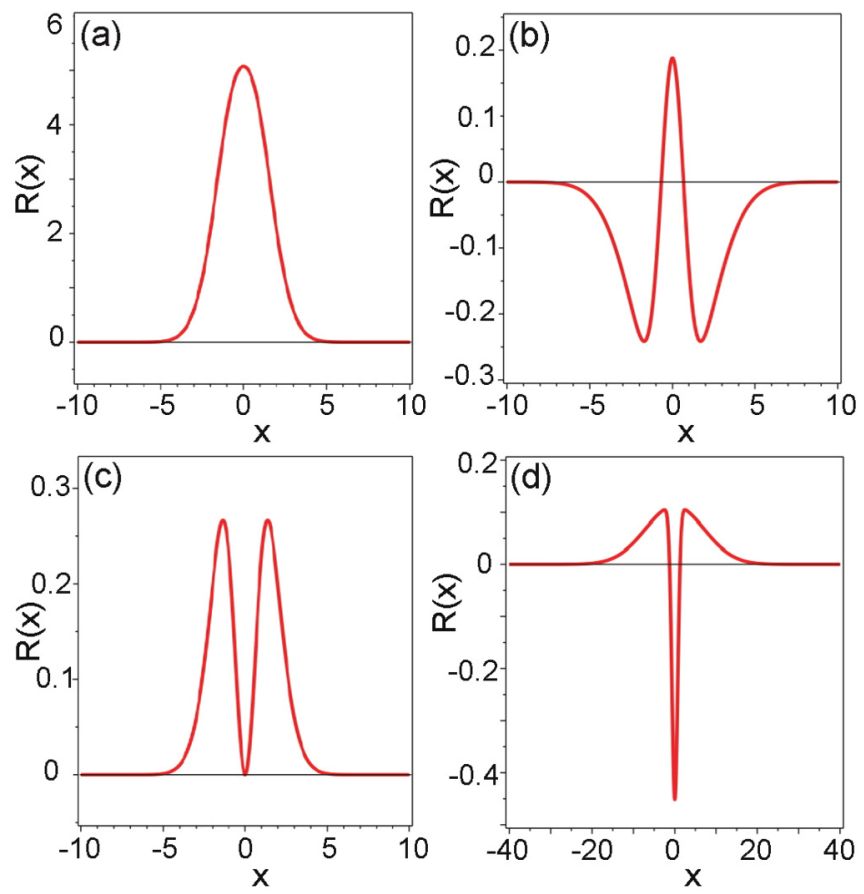

FIG. 1. (Color online) Various profiles of the nonlocal response function $R(x)$ as a function of material parameters: (a) $\alpha_{1}=20$, $\alpha_{2}=-1, \sigma_{1}=2$, and $\sigma_{2}=1$; (b) $\alpha_{1}=2, \alpha_{2}=-1, \sigma_{1}=1$, and $\sigma_{2}=2$; (c) $\alpha_{1}=2, \alpha_{2}=-2, \sigma_{1}=1$, and $\sigma_{2}=3$; and (d) $\alpha_{1}=2$, $\alpha_{2}=-1, \sigma_{1}=10$, and $\sigma_{2}=1$.

competing nonlocal nonlinearities, the shape of the response function will depend strongly on the relative strength of the medium parameters $\alpha_{1}, \alpha_{2}, \sigma_{1}$, and $\sigma_{2}$. The graphs in Fig. 1 illustrate possible profiles of the nonlocal response function as the medium parameters are varied. The response function still decays monotonically for a large value of the spatial coordinate, but in general it can have a rather complex shape. It does not necessarily have extremum in the center and may no longer be sign definite. Since the nonlinear refractive index is determined by convolution of the response function and the beam intensity, the different response functions will affect properties of individual solitons and, more importantly, their interaction.

\section{STATIONARY SOLITONS OF COMPETING NONLOCAL NONLINEARITIES}

We begin with an analytical description of individual solitons in media with competing nonlocal nonlinearities. The nonlocal propagation equation (1) cannot be solved exactly. Instead, we will use an approximation, i.e., the so-called variational technique $[59,60]$, which, although it is only approximate, allows one to obtain an analytical description and physical insight into the properties of the solutions.

Equation (1) can be considered as the Euler-Lagrange variational equation corresponding to the Lagrangian density,

$$
\begin{aligned}
& \mathcal{L}\left(u, u^{*}, u_{z}, u_{z}^{*}, u_{x}, u_{x}^{*}\right) \\
& \quad=\frac{i}{2}\left\{u^{*} \partial_{z} u-u \partial_{z} u^{*}\right\}-\left|\partial_{x} u\right|^{2}+\frac{|u|^{2}}{2} \Delta n(I),
\end{aligned}
$$


with the asterisk denoting a complex conjugate. To find the stationary soliton solutions to Eq. (1) with the variational method, we will use the following Gaussian ansatz:

$$
u(x, z)=A \exp \left[-\frac{(x)^{2}}{2 W^{2}}\right] \exp [i \beta z],
$$

where $A$ is the amplitude of the beam, $W$ is the width, and $\beta$ is the propagation constant.

Now, evaluation of the Lagrangian density [Eq. (3)] with the trial function and integration over $x$ yields the reduced Lagrangian,

$$
\begin{aligned}
\tilde{L}= & -\sqrt{\pi} A^{2} W\left\{\beta+\frac{1}{2} \frac{1}{W^{2}}-\frac{1}{2} \alpha_{1} \frac{A^{2} W}{\sqrt{2 W^{2}+\sigma_{1}^{2}}}\right. \\
& \left.-\frac{1}{2} \alpha_{2} \frac{A^{2} W}{\sqrt{2 W^{2}+\sigma_{2}^{2}}}\right\} .
\end{aligned}
$$

The corresponding equations for soliton parameters are obtained from the Euler-Lagrange equations. In particular, we can derive the following second-order differential equation for soliton width $W$ :

$$
\begin{aligned}
& \frac{d^{2} W}{d z^{2}}+4 \frac{P W}{\sqrt{\pi}}\left\{\frac{\alpha_{1}}{\left(2 W^{2}+\sigma_{1}^{2}\right)^{3 / 2}}+\frac{\alpha_{2}}{\left(2 W^{2}+\sigma_{2}^{2}\right)^{3 / 2}}\right\}-\frac{4}{W^{3}} \\
& =0,
\end{aligned}
$$

where $P=\sqrt{\pi} A^{2} W$ is the soliton power.

Integrating once, we arrive at

$$
\left(\frac{d W}{d z}\right)^{2}+V(W)=E
$$

where

$$
V(W)=-4 \frac{P}{\sqrt{\pi}}\left\{\frac{\alpha_{1}}{\sqrt{2 W^{2}+\sigma_{1}^{2}}}+\frac{\alpha_{2}}{\sqrt{2 W^{2}+\sigma_{2}^{2}}}\right\}+\frac{4}{W^{2}} .
$$

The resulting equation is the equation for an effective particle moving in a potential $V$. Here $E$ is a constant with respect to $z$ and the soliton width $W$ plays the role of the coordinate of an effective particle. The stationary soliton solution, if it exists, corresponds to the particle being located at the bottom of the potential well.

For a stationary solution to exist, the potential must have a local minimum. A simple analysis of Eq. (8) shows that the potential will either decay monotonically or have one or two extrema, depending on the medium parameters.

If it has just one extremum, then this will be a global minimum. If it has two extrema, then the first of them will be the minimum and the second will be a local maximum. In order for the potential to have two extrema, $\alpha_{1}$ must be less than $\left|\alpha_{2}\right|$.

In Fig. 2, we depict two examples of an effective potential [Eq. (8)] for a given power and two sets of medium parameters. In the first case, the $V(x)$ has just a single minimum, while in the second, it features a minimum as well as a local
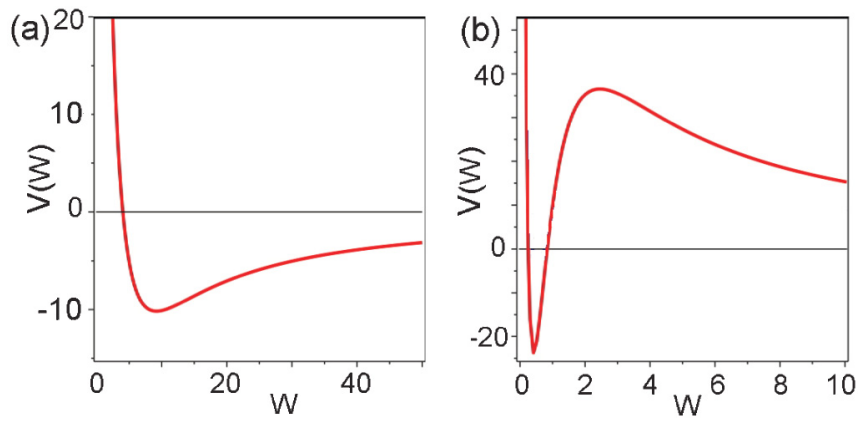

FIG. 2. (Color online) Effective potential [Eq. (8)] for the beam power $P=100$ and material parameters corresponding to (a) $\alpha_{1}=2$, $\alpha_{2}=-1, \sigma_{1}=1$, and $\sigma_{2}=2$ and (b) $\alpha_{1}=2, \alpha_{2}=-2, \sigma_{1}=1$, and $\sigma_{2}=3$.

maximum. We observe that initially the potential decreases with increasing $W$, signifying that a very narrow beam will broaden due to diffraction. The potential reaches a minimum at the stationary width, where the effects of diffraction and nonlinearity exactly cancel each other. It then increases again because the (self-focusing) nonlinearity will dominate diffraction at large widths and focus the beam.

The width of the stationary solution is found from the condition $d V / d W=0$ as the smaller root of the polynomial

$$
\frac{P W_{s}^{4}}{\sqrt{\pi}}\left\{\frac{\alpha_{1}}{\left(2 W_{s}^{2}+\sigma_{1}^{2}\right)^{3 / 2}}+\frac{\alpha_{2}}{\left(2 W_{s}^{2}+\sigma_{2}^{2}\right)^{3 / 2}}\right\}=1 .
$$

Equivalently, the power of the stationary solitons, i.e., the power necessary to have a stationary soliton of width $W_{s}$, is given by

$$
P=\frac{\sqrt{\pi}}{W_{s}^{4}}\left\{\frac{\alpha_{1}}{\left(2 W_{s}^{2}+\sigma_{1}^{2}\right)^{3 / 2}}+\frac{\alpha_{2}}{\left(2 W_{s}^{2}+\sigma_{2}^{2}\right)^{3 / 2}}\right\}^{-1} .
$$

The analysis of Eq. (9) reveals that the stationary solution always exists if $\alpha_{1}>\left|\alpha_{2}\right|$, independently of the power and degree of nonlocality of the constituent nonlinearities. This, of course, means that in media with only a self-focusing nonlocal nonlinearity, a stationary solution always exists. If $\sigma_{1} \geqslant \sigma_{2}$, then one must have $\alpha_{1}>\left|\alpha_{2}\right|$ in order to have a solution to Eq. (9). On the other hand, if $\sigma_{2}>\sigma_{1}$, then a stationary solution may exist even with $\left|\alpha_{2}\right|>\alpha_{1}$. In this case, a necessary, but not sufficient, condition to have a stationary solution is that $\alpha_{1} / \sigma_{1}^{3}>\left|\alpha_{2}\right| / \sigma_{2}^{3}$. In order for the stationary solution to exist, the power must be sufficiently high.

In local media where $\sigma_{1}=\sigma_{2}=0$, Eq. (9) reduces to

$$
W_{s}=\frac{2^{3 / 2} \sqrt{\pi}}{P \alpha},
$$

where $\alpha=\alpha_{1}+\alpha_{2}$. So the stationary width is inversely proportional to the product between the power and nonlinear parameter, i.e., a higher power will lead to a narrower stationary soliton. This is a general property that also holds in the nonlocal case. 
For the stationary solution with $W=W_{s}$ and $A=A_{s}$, the propagation constant $\beta$ is given by the relation

$$
\begin{aligned}
\beta= & B^{2}+A_{s}^{2} W_{s}\left\{\frac{\alpha_{1}}{\sqrt{2 W_{s}^{2}+\sigma_{1}^{2}}}+\frac{\alpha_{2}}{\sqrt{2 W_{s}^{2}+\sigma_{2}^{2}}}\right\} \\
& -\frac{1}{2} A_{s}^{2} W_{s}^{3}\left\{\frac{\alpha_{1}}{\left(2 W_{s}^{2}+\sigma_{1}^{2}\right)^{3 / 2}}+\frac{\alpha_{2}}{\left(2 W_{s}^{2}+\sigma_{2}^{2}\right)^{3 / 2}}\right\} .
\end{aligned}
$$

In the local case, Eq. (12) reduces to

$$
\beta=\left(\alpha_{1}+\alpha_{2}\right) A^{2}\left(\frac{1}{\sqrt{2}}-\frac{1}{2^{5 / 2}}\right) \cong 0.53\left(\alpha_{1}+\alpha_{2}\right) A^{2},
$$

which is in fairly good agreement with the exact formula $d \theta / d z=1 / 2\left(\alpha_{1}+\alpha_{2}\right) A^{2}[2]$.

The important aspect of the stationary solitons is their stability. The shape of the potential $V(x)$ indicates that while the stationary solution which corresponds to a minimum of the potential should be stable, that which corresponds to its maximum should be unstable. Indeed, one can show by using Eqs. (10) and (12) that this conjecture agrees with the well-known Vakhitov-Kolokolov criterion, which relates soliton stability with the relation between soliton power and its propagation constant $P(\beta)$. According to this criterion, solitons are stable (unstable) when $\partial P / \partial \beta>0(<0)$ [61]. To test the stability of variational soliton solutions, we resorted to numerical simulations of the the nonlocal Schrödinger equation Eq. (1). For given sets of medium parameters $\left(\alpha_{j}, \sigma_{j}\right)$ $(j=1,2)$ and power $P$, Eq. (9) is solved, and the stationary width $W_{s}$ as well as the stationary amplitude $A_{s}$ are found. The approximate soliton solution given by Eq. (4) is then used as the initial condition in Eq. (1), and is propagated using a split-step Fourier scheme.

Our simulations confirmed that all stationary solitons corresponding to a minimum of effective potential $V(x)$ are indeed stable. Figure 3(a) shows the propagation of the stationary solution with power $P=100$ corresponding to the medium parameters $\alpha_{1}=2, \alpha_{2}=-1, \sigma_{1}=1$, and $\sigma_{2}=2$. The width of the stationary solution is $W_{s} \approx 0.34$. The soliton is propagated over 20 diffraction lengths, $L_{D}=W^{2} / 2$. The input intensity and nonlinearity-induced refractive index profiles are shown in Fig. 3(b). In the plots, the transverse coordinate $x$ is normalized to the initial width of the soliton.
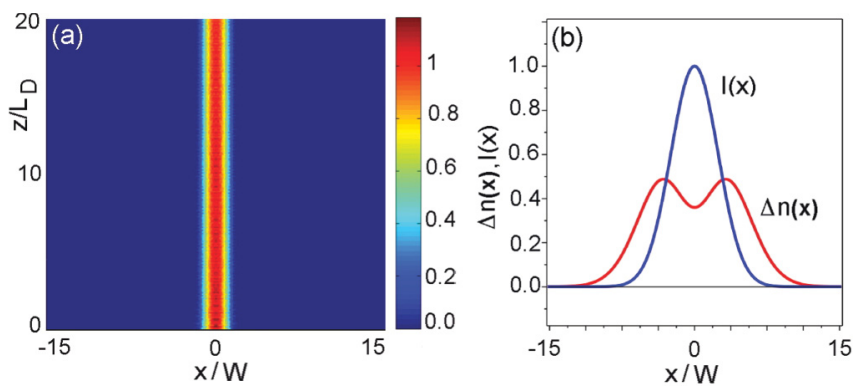

FIG. 3. (Color online) (a) Normalized intensity of stationary soliton with power $P=100$ propagated stably over 20 diffraction lengths. (b) Intensity (blue) and nonlinearity-induced index (red) profiles. The medium parameters are $\alpha_{1}=2, \alpha_{2}=-1, \sigma_{1}=1$, and $\sigma_{2}=2$.
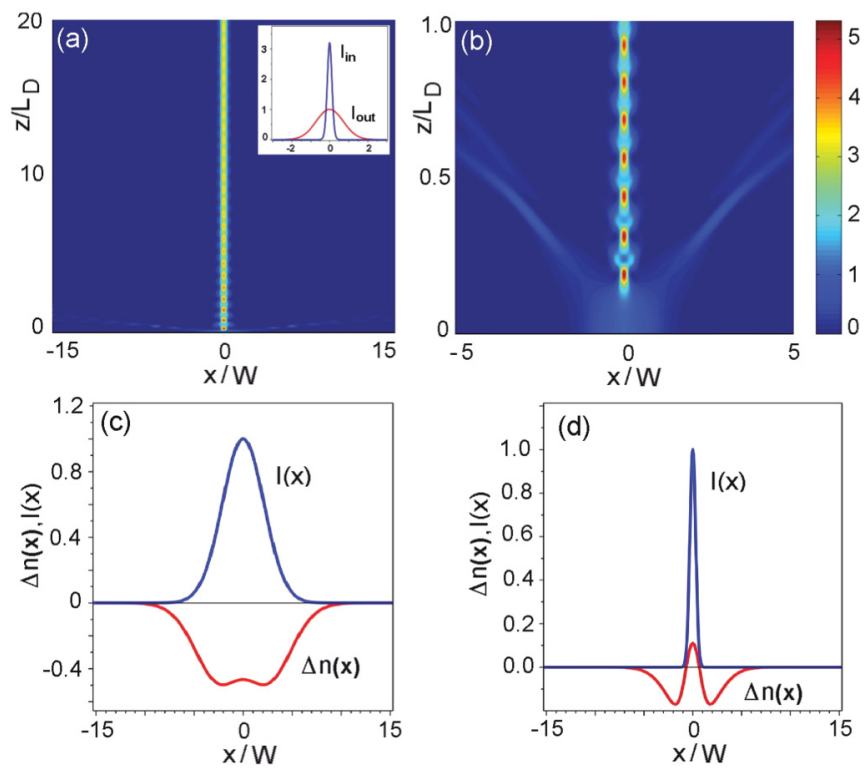

FIG. 4. (Color online) (a), (b) Evolution of beam width: the initial beam width $W$ exceeding the value corresponding to the maximum of an effective potential in Fig. 2(b); (b) zoom of graph (a). (c) Initial and (d) final transverse intensity and refractive index profiles. Here the initial power of the beam $P=100$. The medium parameters are $\alpha_{1}=2, \alpha_{2}=-2, \sigma_{1}=1$, and $\sigma_{2}=3$.

The form of the effective potential depicted in Fig. 2(b) suggests that if the initial width of the beam is larger than the value for the second extremum, then the width will increase continuously during propagation. In Figs. 4(a) and 4(b), we depict propagation of the optical beam of initial width $W=3$ and power $P=100$ over 20 diffraction lengths. The inset shows the initial and final intensity profiles. Clearly, the dynamics of the beam is rather unexpected. Instead of spreading, the initially broad beam emits an excess of power in the form of linear waves and subsequently undergoes dynamic transition into a much narrower, higher intensity beam. In fact, it turns out that the final beam is actually a soliton with a new amplitude, $A \approx \sqrt{62}$, width $W \approx 0.51$, and power of $P \approx 56$. These parameters correspond to stationary soliton solutions described by the reduced Lagrangian. The observed behavior is quite unique. To explain it, the initial refractive index distribution [Fig. 4(c)] is fundamental. Sufficiently far away from the center, the refractive index is monotonically increasing to zero. Thus the refractive index at the periphery is larger than at the center, and light will leak into the surrounding areas during propagation. However, in the center it is increasing too, so the refractive index at $x=0$ is slightly larger than in the near neighborhood. Strictly speaking, such a structure is not a waveguide because the high refractive index at the beam wings will prevent the asymptotic decay of a wave amplitude, but the narrow "channel" gives rise to local focusing of the beam. The combination of these two mechanisms modifies the intensity distribution and, subsequently, the refractive index structure, and thereby increases coupling into the narrow channel. Ultimately, a proper waveguide structure is formed and so power will couple into the waveguide, leading finally to the formation of a proper stable soliton. 
We should note that if the initial width is sufficiently large, then the beam simply diffracts, and no solitons are formed.

\section{VARIATIONAL APPROACH TO COHERENT SOLITON INTERACTION}

To describe the coherent interaction of two well-separated, identical solitons with the variational method, we will employ a general two-soliton ansatz,

$$
\begin{aligned}
u(x, z)= & u_{1}(x, z)+u_{2}(x, z) \\
= & A \exp \left[-\frac{(x-\xi)^{2}}{2 W^{2}}\right] \exp [i \theta+i B(x-\xi)] \\
& +A \exp \left[-\frac{(x+\xi)^{2}}{2 W^{2}}\right] \exp [i \phi+i \theta-i B(x+\xi)],
\end{aligned}
$$

where $\phi$ denotes an initial phase difference between solitons. Parameter $B$ represents the transverse "velocity" of the soliton. In fact, one can show that $2 B=d \xi / d z$. For simplicity, we only consider the situation where the two solitons either move towards or away from each other, so we use $B_{1}=-B_{2}=B$.

The total intensity $|u(x, z)|^{2}$ reads

$$
\begin{aligned}
|u(x, z)|^{2}= & \left|u_{1}(x, z)\right|^{2}+\left|u_{2}(x, z)\right|^{2} \\
& +2\left|u_{1}(x, z)\right|\left|u_{2}(x, z)\right| \cos [\phi-2 B x] .
\end{aligned}
$$

The oscillating term represents variation of the relative phase of two solitons. In the highly nonlocal limit $\left(\sigma_{j} \rightarrow \infty\right)$, its contribution lies outside the bandwidth of the Fouriertransformed response function, and the resulting medium response is phase independent, i.e., incoherent. Even if the solitons interact coherently, the nonlocality still gives rise to a smoothed-out nonlinear refractive index, which depends primarily on the incoherent sum of the soliton amplitudes, and hence entails incoherentlike soliton interactions [34].

For given medium parameters $\left(\alpha_{j}, \sigma_{j}\right)(j=1,2)$ and power of the individual solitons $P=\sqrt{\pi} A^{2} W$, the stationary width and amplitude are determined by Eq. (9). From now on, we denote the width and amplitude by $W_{s}$ and $A_{s}$, respectively, to stress that they are given by their stationary values. We will consider only weakly interacting solitons, hence we can assume that their amplitude $A$ and width $W$ are equal to their stationary values. However, we keep the $z$ dependence in the soliton phase $\theta=\theta(z)$ and velocity $B=B(z)$.

The Lagrangian density of the system, given by Eq. (3), is evaluated on the two-soliton trial function, and integrated over $x$. After lengthy calculations, assuming well-separated solitons, we obtain the reduced Lagrangian

$$
\begin{aligned}
\tilde{L}= & -2 \sqrt{\pi} A_{s}^{2} W_{s}\left\{\left(\frac{d \theta}{d z}-B \frac{d \xi}{d z}+\frac{1}{2} \frac{1}{W_{s}^{2}}+B^{2}\right)\right. \\
& +\left(\frac{d \theta}{d z}-\xi \frac{d B}{d z}+\frac{1}{2} \frac{1}{W_{s}^{2}}-\frac{\xi^{2}}{W_{s}^{4}}\right) \exp \left[-\frac{\xi^{2}}{W_{s}^{2}}\right] \cos [\phi] \\
& \left.-\sum_{i=1,2} \alpha_{i} \frac{A_{s}^{2} W_{s}}{2 \sqrt{2 W_{s}^{2}+\sigma_{i}^{2}}}\left(1+\exp \left[-4 \frac{\xi^{2}}{2 W_{s}^{2}+\sigma_{1}^{2}}\right]\right)\right\} .
\end{aligned}
$$
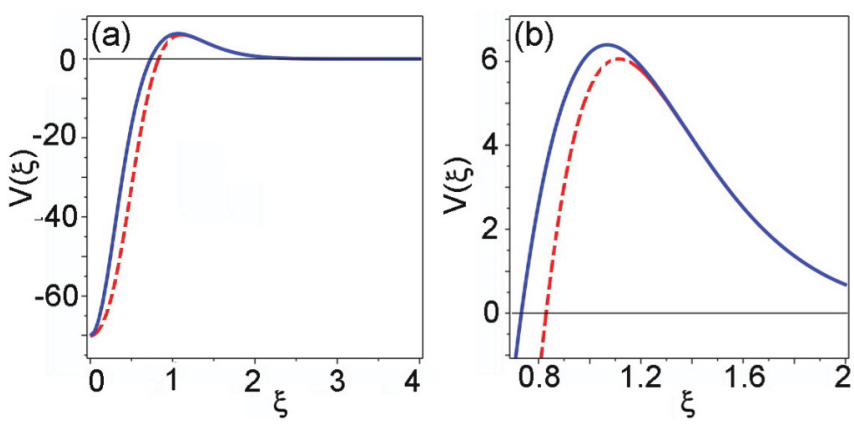

FIG. 5. (Color online) (a) Effective potential $V$ for in-phase (dashed red line) and out-of-phase (solid blue line) interacting solitons corresponding to the parameters $P=50, \alpha_{1}=2, \alpha_{2}=-1, \sigma_{1}=1$, and $\sigma_{2}=2$. (b) Zoom from (a).

From the ensuing Euler-Lagrange equations, we arrive at the following effective particle equation:

$$
\frac{d^{2} \xi}{d z^{2}}=-\frac{d^{2} V(\xi)}{d \xi}
$$

where the effective potential is given as

$$
\begin{aligned}
V(\xi)= & -4 \frac{\xi^{2}}{W_{s}^{4}} \exp \left[-\frac{\xi^{2}}{W_{s}^{2}}\right] \cos \phi \\
& -2 \alpha_{1} \frac{P}{\sqrt{\pi}} \sum_{i=1,2} \frac{1}{\sqrt{2 W_{s}^{2}+\sigma_{i}^{2}}} \exp \left[-4 \frac{\xi^{2}}{2 W_{s}^{2}+\sigma_{i}^{2}}\right],
\end{aligned}
$$

and the spatial soliton separation plays the role of the particle coordinate. The parameter $P$ denotes the power of the individual soliton, $P=\sqrt{\pi} A_{s}^{2} W_{s}$.

Notice that only the first term in the potential depends on the relative phase between solitons. For sufficiently high degrees of nonlocality or powers, this term becomes negligible and inphase $(\phi=0)$ and out-of-phase $(\phi=\pi)$ solitons will interact similarly, i.e., the interaction is incoherent.

Figure 5(a) shows the potential as a function of $\xi$ for both inphase and out-of-phase interacting solitons of power $P=50$. The medium parameters are $\alpha_{1}=2, \alpha_{2}=-1, \sigma_{1}=1$, and $\sigma_{2}=2$, resulting in the stationary width $W_{s} \approx 0.42$. We observe that the potential increases from a minimum value until it reaches a maximum and then decays monotonically to zero. An increasing potential corresponds to attraction between the two solitons, while a decreasing potential corresponds to repulsion. The potential does not form a well, and thereby does not predict a stable stationary state, where the two solitons propagate side by side with no net force between them. At the separation corresponding to $d V / d \xi=0$, the force between the solitons is zero, but the state will not be stable. The point marks the transition between an attractive and a repulsive potential, and will be denoted $\xi_{t}$. In the zoom shown in Fig. 5(b), we see that the transition between repulsion and attraction occurs at different separations for in-phase and out-of-phase solitons.

In order to understand why two solitons attract each other for sufficiently small spatial separations and repel when the separation is increased, we need to consider the nonlinearityinduced refractive index change $\Delta n$. Figures 6 (a) and 6(b) show the intensity profile and the refractive index for two 

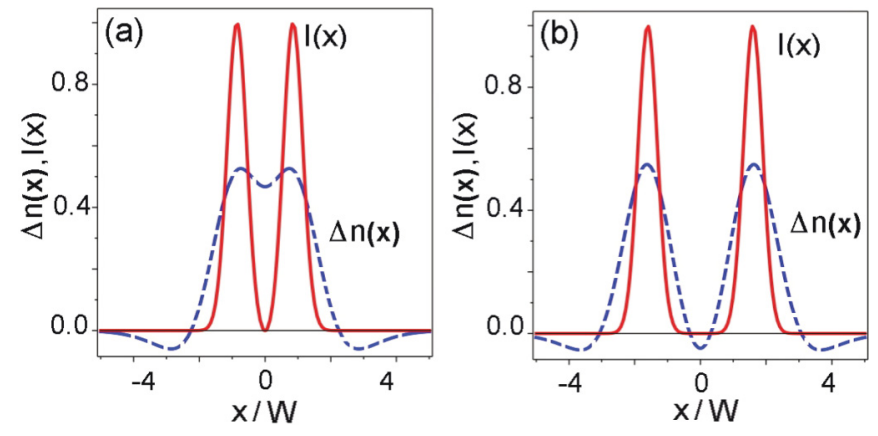

FIG. 6. (Color online) Intensity (solid red line) and nonlinear refractive index change (dashed blue line) of two out-of-phase solitons with spatial separation of (a) $0.8 \xi_{t}$ and (b) $1.5 \xi_{t}$, where $\xi_{t} \approx 0.44$. The parameters are $P=50, \alpha_{1}=2, \alpha_{2}=-1, \sigma_{1}=1$, and $\sigma_{2}=2$.

out-of-phase solitons with spatial separation $\xi=0.8 \xi_{t}$ and $\xi=1.5 \xi_{t}$, respectively, in a medium with $\alpha_{1}>\left|\alpha_{2}\right|$.

When $\xi<\xi_{t}$, the nonlinear refractive index $(\Delta n)$ increases in the region between solitons, leading to their attraction. When the separation is increased and $\xi>\xi_{t}$, the index $\Delta n$ drops, resulting in the solitons moving away from each other in what appears as repulsion.

Nonlocal solitons experience the presence of each other even if they are launched with a large separation. The nonlocality originates a refractive index distribution wide enough to connect the two solitons, even though the field overlap is almost zero [Fig. 6(b)]. Hence, two well-separated solitons in nonlocal media can interact, while in local Kerr media, they propagate independently because of the localized index distribution.

Figure 7(a) depicts the effective potential for the parameters $P=50, \alpha_{1}=2, \alpha_{2}=-1, \sigma_{1}=2$, and $\sigma_{2}=1$. We observe that for in-phase interacting solitons, the potential is always attractive, and so the solitons will never repel. On the other hand, if we look at the zoom shown in Fig. 7(b), we see that the potential is in fact changing between being attractive and being repulsive for out-of-phase solitons. So they will repel for sufficiently large separations.

A careful analysis of Eq. (18) [together with Eq. (9)] reveals that if $\alpha_{1}>\left|\alpha_{2}\right|$ and $\sigma_{1}>\sigma_{2}$, then the potential will always be attractive for the interaction between in-phase solitons, while it
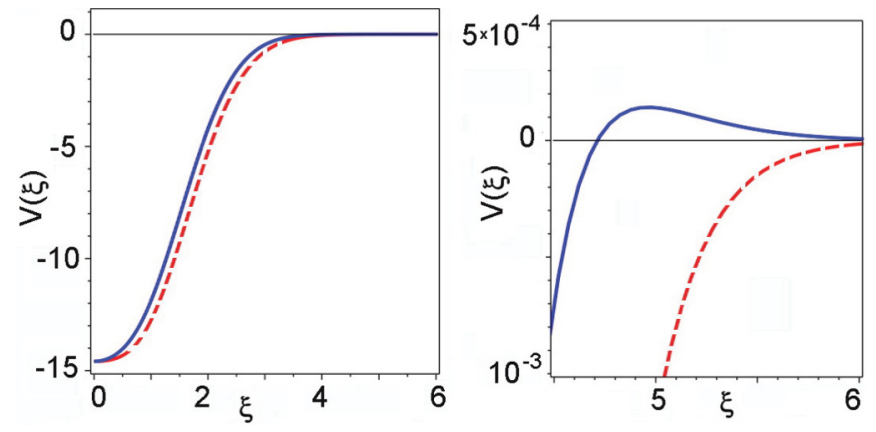

FIG. 7. (Color online) (a) Effective potential $V(\xi)$ for in-phase (dashed red line) and out-of-phase (solid blue line) interacting solitons corresponding to the parameters $P=50, \alpha_{1}=2, \alpha_{2}=-1, \sigma_{1}=2$, and $\sigma_{2}=1$. (b) Zoom from (a).
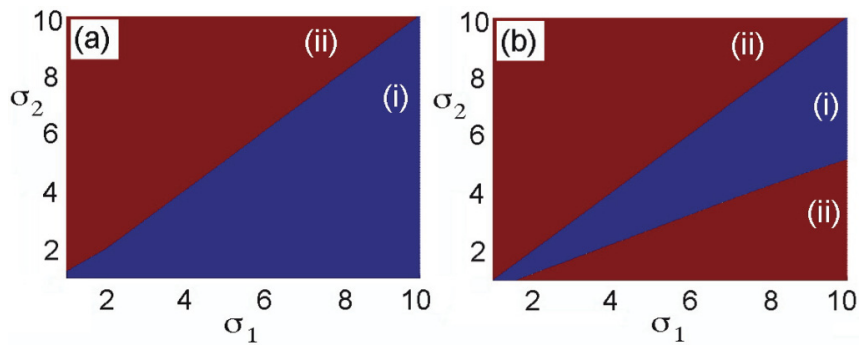

FIG. 8. (Color online) Properties of effective potential $V(\xi)$ for (a) in-phase and (b) out-of-phase interacting solitons. Blue (i) indicates an always attractive potential, while red (ii) indicates that the potential is either attractive or repulsive depending on the spatial separation. The parameters are $P=10, \alpha_{1}=2$, and $\alpha_{2}=-1$.

may be both attractive and repulsive for the interaction between out-of-phase solitons. On the other hand, if $\sigma_{2}>\sigma_{1}$, then it will always be both attractive and repulsive for the interaction between out-of-phase solitons, while it may be only attractive for the interaction between in-phase solitons. By being both attractive and repulsive, we mean that the potential has a transition point, such that for $\xi<\xi_{t}$, it is attractive, while for $\xi>\xi_{t}$, it is repulsive.

These properties of the potential are illustrated in Fig. 8 for given parameters $P, \alpha_{1}$, and $\alpha_{2}$. Here the blue color indicates an always attractive potential, while the red color indicates an either attractive or repulsive potential, depending on the spatial separation of the interacting solitons.

In the regime $\left|\alpha_{2}\right|>\alpha_{1}$, the potential is either attractive or repulsive, depending on the spatial separation of the interacting solitons. We remember that in this regime, one must have $\sigma_{2}>\sigma_{1}$ for a stationary solution to exist. If the first term in Eq. (18) is negligible (the interaction is incoherent), then we can more generally say that for $\sigma_{1}>\sigma_{2}$, the potential is only attractive, while for $\sigma_{2}>\sigma_{1}$, it is either attractive or repulsive depending on the separation between the interacting solitons.

In Fig. 9(a), we illustrate the dependence of the transition separation, $\xi_{t}$, on the soliton power for both in-phase and outof-phase interacting solitons. In the former case, the transition separation decreases monotonically with increasing power, whereas in the latter case, there is a local minimum in $\xi_{t}(P)$. When the power is sufficiently high, the phase-dependent term
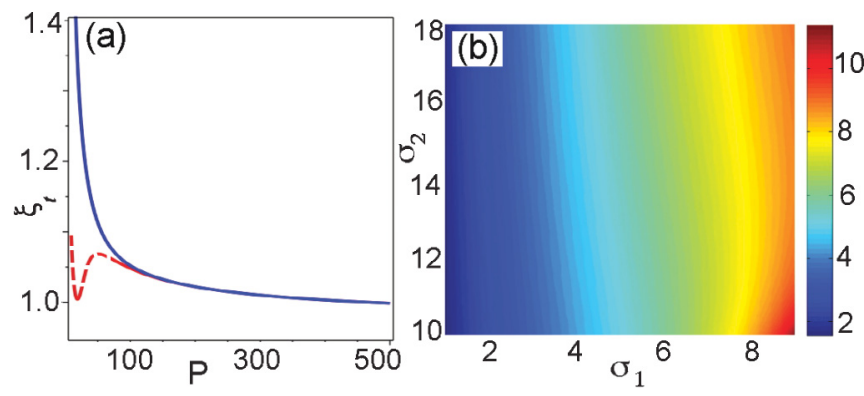

FIG. 9. (Color online) (a) Transition separation as a function of power $(P \in[10,500])$ for $\phi=0$ (solid blue line) and $\phi=\pi$ (dashed red line). The medium parameters are $\alpha_{1}=2, \alpha_{2}=-1, \sigma_{1}=1$, and $\sigma_{2}=2$. (b) Transition separation as a function of width of nonlocal nonlinearities for $\phi=\pi$ and $P=50$. The medium parameters are $\alpha_{1}=2$ and $\alpha_{2}=-1$. 
in Eq. (18) becomes negligible, and the transition separation for in-phase and out-of-phase interacting solitons is almost identical. The observed decrease in $\xi_{t}$ with increasing $P$ is due to the fact that a higher power of the solitons leads to a lower (normalized) intensity in the overlap region, and thus a lower (normalized) refractive index in the center between the solitons.

Figure 9(b) shows the transition separation for out-of-phase interacting solitons as a function of $\sigma_{1}$ and $\sigma_{2}$ for given parameters $P, \alpha_{1}$, and $\alpha_{2}$. Evidently, $\xi_{t}$ simply increases with increasing $\sigma_{1}$, whereas the dependence on $\sigma_{2}$ is nontrivial. For small $\sigma_{1}, \xi_{t}$ increases with increasing $\sigma_{2}$, but for large $\sigma_{1}$, $\xi_{t}\left(\sigma_{2}\right)$ is nonmonotonic.

It is important to note that according to the potential in Eq. (18), in-phase solitons will always be attracting in media with only a self-focusing nonlocal nonlinearity. Out-of-phase solitons may be repelling, but if the phase-dependent term in the potential can be left out of account, out-of-phase solitons will always be attracting too. This predicted behavior is in agreement with the numerical results in [34]. Furthermore, it has been observed experimentally and even exploited to build all-optical logic gates [33]. The fact that interacting solitons may repel for sufficiently large spatial separations is a pure consequence of the competing self-defocusing nonlinearity. The results are, of course, only valid for separations sufficiently larger than the width of the individual solitons.

\section{NUMERICAL SIMULATIONS OF COHERENT SOLITON INTERACTION}

In this section, we investigate the validity of the variational results obtained in Sec. IV by using numerical simulations. For given sets of medium parameters $\left(\alpha_{j}, \sigma_{j}\right)(j=1,2)$ and power $P$, Eq. (9) is solved and the stationary width $W_{s}$ and amplitude $A_{s}$ are found. When these parameters are known, the transition separation $\xi_{t}$, if it exists, can be determined by solving $d V / d \xi=0$ for $\xi$. The two-soliton ansatz [Eq. (14)] is then used as the initial condition in Eq. (1), and is propagated using a split-step Fourier scheme.

The potential shown in Fig. 5 shows that for the given medium parameters $\left(\alpha_{1}=2, \alpha_{2}=-1, \sigma_{1}=1\right.$, and $\left.\sigma_{2}=2\right)$, stationary solitons, with individual power $P=50$, will either attract or repel each other, depending on their initial spatial separation. This applies to both in-phase and out-of-phase interacting solitons, but the transition between attraction and repulsion occurs at different separations.

Figure 10 shows the propagation of two in-phase solitons with initial spatial separation $\xi=\xi_{t}$. For the given power and medium parameters, the transition separation is found to be $\xi_{t} \approx 1.11$. The initial transverse velocity $B$ of the solitons is zero, as it is in all of the cases studied below. We observe attraction between the two solitons and, eventually, collision. If the initial separation between them is increased to $\xi=1.02 \xi_{t}$, then, as Fig. 10(b) shows, the solitons now repel each other, as predicted by the variational calculations. The solitons will continue to move apart from each other. The initial separation of the solitons has to be increased to $\sim 1.02 \xi_{t}$ in order to observe repulsion, due to the inaccuracy of the approximate variational results.

Figure 11 shows the same scenario for out-of-phase interacting solitons. In the simulation shown in the first of
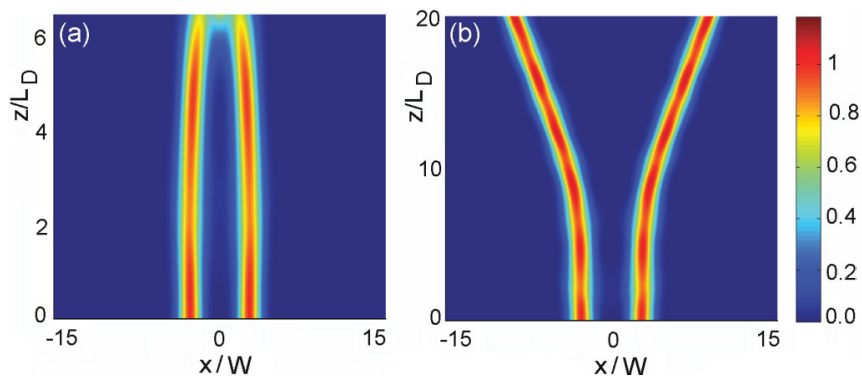

FIG. 10. (Color online) Trajectories of in-phase interacting solitons with power $P=50$ and initial separation (a) $\xi=\xi_{t}$ and (b) $\xi=1.02 \xi_{t}$, where $\xi_{t} \approx 1.11$. The medium parameters are $\alpha_{1}=2$, $\alpha_{2}=-1, \sigma_{1}=1$, and $\sigma_{2}=2$.

these figures, the initial separation is $\xi=\xi_{t}$, and we observe that the solitons attract each other. When the initial separation is increased to $\xi=1.02 \xi_{t}$, the solitons repel, as shown in the latter figure. For out-of-phase interacting solitons, the transition separation is found to be $\xi_{t} \approx 1.07$.

If the in-phase interacting solitons in Fig. 10 are propagated further, they collide, as depicted in Fig. 12(a). Due to the nonintegrability of the governing propagation equation (1), the collision is inelastic. We observe that when the two solitons collide, the intensity increases in the overlap region and power is irradiated as diffractive waves. The solitons intersect quasiperiodically during propagation, until they eventually fuse. On the other hand, if we propagate the two out-of-phase interacting solitons over long distance, they never overlap. They approach each other, but when they come sufficiently close, they start repelling, as shown in Fig. 12(b), and so they never touch. As the spatial separation is increased, they start attracting again and we observe a quasiperiodical behavior.

It was noted in Sec. IV that the assumptions imposed to derive Eq. (16) boil down to having sufficiently high power of the interacting solitons. Thus, the assumptions are even better satisfied for higher powers. Indeed, our simulations of the interaction of two out-of-phase solitons with power $P=500$ show that the transition separation predicted by the variational calculations is within $0.5 \%$ of the exact numerical value, and thereby the accuracy of the variational results increases with increasing power.

In the derivation of the potential in Eq. (18), it was assumed that the separation of the two interacting solitons is much larger than the width of the solitons themselves. Under
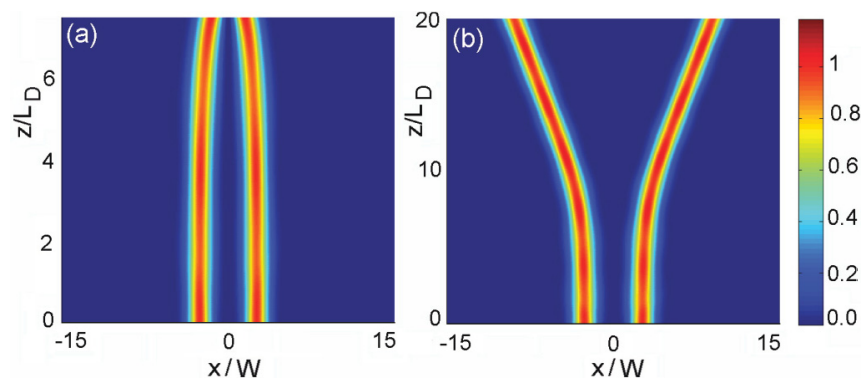

FIG. 11. (Color online) Trajectories of interacting out-of-phase solitons with power $P=50$ and initial separation (a) $\xi=\xi_{t}$ and (b) $\xi=1.02 \xi_{t}$, where $\xi_{t} \approx 1.07$. The medium parameters are $\alpha_{1}=2$, $\alpha_{2}=-1, \sigma_{1}=1$, and $\sigma_{2}=2$. 

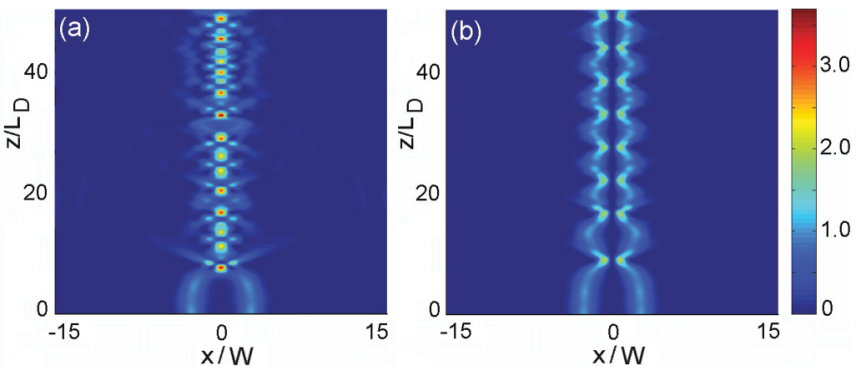

FIG. 12. (Color online) Trajectories of interacting in-phase solitons with power $P=50$ and initial separation (a) $\xi=\xi_{t} \approx 1.11$ and (b) $\xi=\xi_{t} \approx 1.07$. The medium parameters are $\alpha_{1}=2, \alpha_{2}=-1$, $\sigma_{1}=1$, and $\sigma_{2}=2$.

this assumption, the variational results will never be able to describe the well-known stationary state of two out-of-phase solitons, i.e., the so-called dipole-mode soliton [41,62], with a spatial separation on the order of the width of the solitons.

To find the stationary dipole state, we will assume that all soliton parameters except $\theta$ in the ansatz [Eq. (14)] are constants with respect to $z$. Then one can show that the EulerLagrange equations for the three parameters $A, W$, and $\xi$ yield the single equation which determines the dipole soliton solution,

$$
\begin{aligned}
& \frac{1}{2 W_{s}}-\left\{\frac{1}{2 W_{s}}-\frac{\xi_{s}^{2}}{W_{s}^{3}}\right\} \exp \left[\frac{-\xi_{s}^{2}}{W_{s}^{2}}\right] \\
& -\sum_{i=1,2} \frac{2 \alpha_{i} A_{s}^{2} W_{s}^{4}}{\left(2 W_{s}^{2}+\sigma_{i}^{2}\right)^{3 / 2}}\left\{1+\exp \left[-\frac{4 \xi_{s}^{2}}{2 W_{s}^{2}+\sigma_{i}^{2}}\right]\right. \\
& \left.-4 \exp \left[\frac{-\xi_{s}^{2}}{W_{s}^{2}}-\frac{\xi_{s}^{2}}{2 W_{s}^{2}+\sigma_{i}^{2}}\right]+2 \exp \left[\frac{-2 \xi_{s}^{2}}{W_{s}^{2}}\right]\right\} \\
& -2 \sum_{i=1,2} \frac{\alpha_{i} A_{s}^{2} W_{s}^{2}}{\sqrt{2 W_{s}^{2}+\sigma_{i}^{2}}}\left\{-\frac{\xi_{s}^{2}}{2 W_{s}^{2}+\sigma_{i}^{2}}+2 \frac{W_{s}^{2} \xi^{2}}{\left(2 W_{s}^{2}+\sigma_{i}^{2}\right)^{2}}\right\} \\
& \quad \times\left\{\exp \left[\frac{-4 \xi_{s}^{2}}{2 W_{s}^{2}+\sigma_{i}^{2}}\right]+\exp \left[-\frac{\xi_{s}^{2}}{W_{s}^{2}}-\frac{\xi_{s}^{2}}{2 W_{s}^{2}+\sigma_{i}^{2}}\right]\right\}=0 .
\end{aligned}
$$

By using the stationary values of $W_{s}$ and $A_{s}$, determined by Eq. (9) for given power $P$ and $\left(\alpha_{j}, \sigma_{j}\right)(j=1,2)$, Eq. (18) can be solved numerically for $\xi_{s}$.

For the medium parameters $\alpha_{1}=2, \alpha_{2}=-1, \sigma_{1}=1$, and $\sigma_{2}=2$, and power $P=50$, Eq. (18) yields the two solutions $\xi=\xi_{t} \approx 1.03$ and $\xi=\xi_{s} \approx 0.44$. The first one is the transition point separating attraction and repulsion, as discussed earlier. The second solution $\xi=\xi_{s}$ represents separation between constituent solitons forming the dipole-mode state. Figure 13(a) shows the input and output intensity profiles of the stationary propagation (over 50 diffraction lengths) of the dipole soliton consisting of two out-of-phase solitons with initial separation $\xi=\xi_{s}$, for the given parameters $W_{s} \approx 0.42$. Figure 13(b) show the scenario when the initial separation is slightly larger than the stationary value $\xi=1.1 \xi_{s}$. We now observe that while the separation between the constituent solitons oscillates during propagation, the dipole state remains stable.
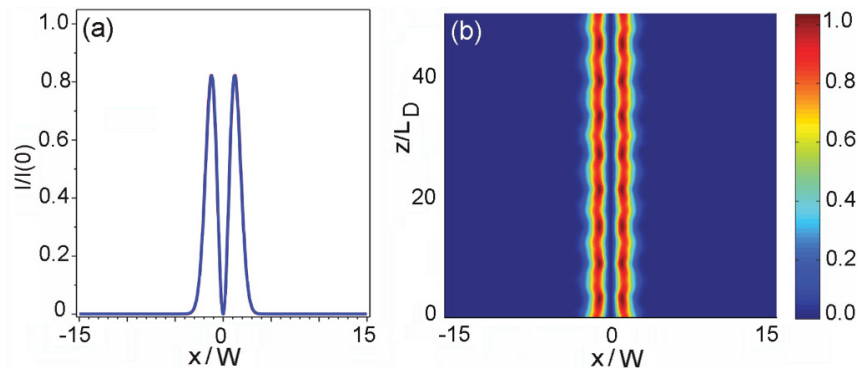

FIG. 13. (Color online) (a) Intensity profile of the dipole-mode nonlocal soliton (or bound state of two out-of-phase solitons). Separation between constituent solitons $\xi_{s} \approx 0.44$. (b) Dynamics of the dipole soliton initially perturbed by increasing the separation between solitons to $\xi=1.1 \xi_{s}$. The parameters are $P=50, \alpha_{1}=2$, $\alpha_{2}=-1, \sigma_{1}=1$, and $\sigma_{2}=2$.

\section{VARIATIONAL APPROACH TO INCOHERENT SOLITON INTERACTION}

In this chapter, we consider the interaction between two mutually incoherent one-dimensional (1D) solitons in media with competing nonlocal Kerr nonlinearities. The two solitons interact only through the nonlinearity, which depends on the superposition of the individual intensities, rather than the amplitudes, and so is independent of the relative phase between the solitons. The interaction can be described by the following set of two coupled nonlocal NLS equations $[56,59,63]$ :

$$
\begin{aligned}
& i \frac{\partial u}{\partial z}+\frac{\partial^{2} u}{\partial x^{2}}+\Delta n u=0, \\
& i \frac{\partial v}{\partial z}+\frac{\partial^{2} v}{\partial x^{2}}+\Delta n v=0,
\end{aligned}
$$

where $u$ and $v$ represent the amplitudes of the solitons. The nonlinear refractive index is given by

$$
\Delta n=\int_{-\infty}^{\infty} R\left(x-x^{\prime}\right)\left\{\left|u\left(x^{\prime}, z\right)\right|^{2}+\left|v\left(x^{\prime}, z\right)\right|^{2}\right\} d x^{\prime},
$$

where $R$ is the full nonlocal response function of the medium,

$$
R(x)=\alpha_{1} R_{1}(x)+\alpha_{2} R_{2}(x) .
$$

Equations (19) and (20) can be considered as the EulerLagrange variational problem corresponding to the following Lagrangian density:

$$
\begin{aligned}
\mathcal{L}(u, v, \ldots)= & -\operatorname{Im}\left\{u^{*} \partial_{z} u+v^{*} \partial_{z} v\right\}-\left(\left|\partial_{x} u\right|^{2}+\left|\partial_{x} v\right|^{2}\right) \\
& +\frac{|u|^{2}+|v|^{2}}{2} \Delta n(I),
\end{aligned}
$$

where the asterisk denotes the complex conjugate.

For simplicity, we will consider the interaction of two identical, well-separated, and hence weakly interacting solitons, which will be represented by the following Gaussian trial functions:

$$
\begin{aligned}
& u(x, z)=A \exp \left[-\frac{(x-\xi)^{2}}{2 W^{2}}\right] \exp \left[i \theta_{1}+i B(x-\xi)\right], \\
& v(x, z)=A \exp \left[-\frac{(x+\xi)^{2}}{2 W^{2}}\right] \exp \left[i \theta_{2}-i B(x+\xi)\right],
\end{aligned}
$$


where $A(z)$ is the soliton amplitude, $W(z)$ is the soliton width, and $\xi(z)$ are the spatial positions of the individual solitons. $\theta_{1}(z), \theta_{2}(z)$ and $B(z)$ represent soliton phase, phase tilt, respectively.

For given medium parameters $\left(\alpha_{j}, \sigma_{j}\right)(j=1,2)$ and power of the individual solitons $P=\sqrt{\pi} A^{2} W$, the stationary width and amplitude are determined by Eq. (9). We will from now on denote the width and amplitude by $W_{s}$ and $A_{s}$, respectively, to stress that they are given by their stationary values.

We evaluate the Lagrangian density of the system, given by Eq. (23), on the trial functions, and integrate over $x$, to obtain the reduced Lagrangian

$$
\begin{aligned}
\tilde{L}= & -2 \sqrt{\pi} A_{s}^{2} W_{s}\left\{\frac{\partial \theta}{\partial z}-B \frac{\partial \xi}{\partial z}+\frac{1}{2} \frac{1}{W_{s}^{2}}+B^{2}\right\} \\
& +\sqrt{\pi} A_{s}^{4} W_{s}^{2} \sum_{j=1}^{2} \frac{\alpha_{j}}{\sqrt{2 W_{s}^{2}+\sigma_{j}^{2}}}\left\{1+\exp \left[-4 \frac{\xi^{2}}{2 W_{s}^{2}+\sigma_{j}^{2}}\right]\right\},
\end{aligned}
$$

where $2 \theta=\theta_{1}+\theta_{2}$.

From the ensuing Euler-Lagrange equations for the reduced Lagrangian, we can derive the following equation for soliton position $\xi$ :

$$
\frac{d^{2} \xi}{d z^{2}}+\sum_{j=1}^{2} 8 \alpha_{j} \frac{A_{s}^{2} W_{s} \xi}{\left(2 W_{s}^{2}+\sigma_{j}^{2}\right)^{3 / 2}} \exp \left[-4 \frac{\xi^{2}}{2 W_{s}^{2}+\sigma_{j}^{2}}\right]=0 .
$$

Here $P_{2}=2 P$, where $P=\sqrt{\pi} A_{s}^{2} W_{s}$ is the power of the individual solitons. This equation can be then transformed into the following relation for an effective particle:

$$
\frac{d^{2} \xi}{d z^{2}}=-\frac{d V(\xi)}{d \xi}
$$

where

$$
\begin{aligned}
V(\xi)= & -2 \alpha_{1} \frac{P}{\sqrt{\pi}} \frac{1}{\sqrt{2 W_{s}^{2}+\sigma_{1}^{2}}} \exp \left[-4 \frac{\xi^{2}}{2 W_{s}^{2}+\sigma_{1}^{2}}\right] \\
& -2 \alpha_{2} \frac{P}{\sqrt{\pi}} \frac{1}{\sqrt{2 W_{s}^{2}+\sigma_{2}^{2}}} \exp \left[-4 \frac{\xi^{2}}{2 W_{s}^{2}+\sigma_{2}^{2}}\right]
\end{aligned}
$$

is an effective potential, and the spatial soliton separation plays the role of the position of the particle.

Figure 14(a) shows the potential as a function of $\xi$ when $\alpha_{1}>\left|\alpha_{2}\right|$ for both $\sigma_{2}>\sigma_{1}$ and $\sigma_{1}>\sigma_{2}$. An increasing potential corresponds to attraction between the two solitons, while a decreasing potential corresponds to repulsion. We observe that when $\sigma_{2}>\sigma_{1}$, the potential is attractive for small separations, but becomes repulsive when the separation is increased. At the separation corresponding to $d V / d \xi=0$, the force between the solitons is zero, but the corresponding state is not stable. The point marks the transition between an attractive and a repulsive potential, and will be denoted $\xi_{t}$. When $\sigma_{1}>\sigma_{2}$, the potential is always attractive, independent of the spatial separation of the interacting solitons.
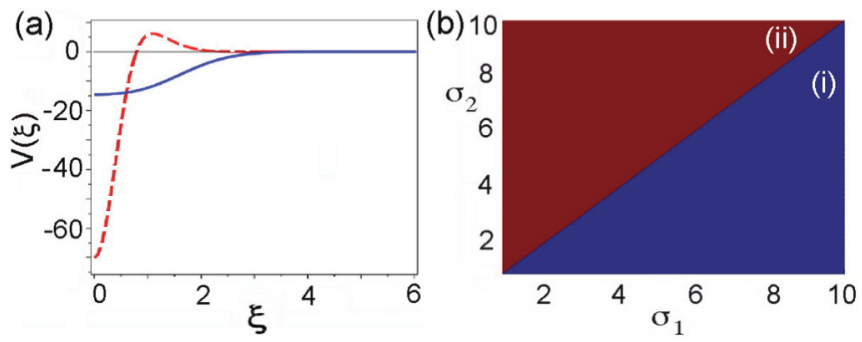

FIG. 14. (Color online) (a) Effective potential of incoherently interacting solitons for $P=50, \alpha_{1}=2$, and $\alpha_{2}=-1$. Dashed red (solid blue) curve corresponds to $\sigma_{1}=1, \sigma_{2}=2\left(\sigma_{1}=2, \sigma_{2}=1\right)$. (b) Properties of the potential for $\alpha_{1}>\left|\alpha_{2}\right|$. Blue (i) indicates an always attractive potential, while red (ii) indicates that the potential is either attractive or repulsive depending on the spatial separation of the solitons.

The transition point $\xi_{t}$ can be found by analytically solving the equation $d V / d \xi=0$ to get

$\xi_{t}=\frac{1}{2} \sqrt{\frac{\left(2 W_{s}^{2}+\sigma_{1}^{2}\right)\left(2 W_{s}^{2}+\sigma_{2}^{2}\right)}{\sigma_{2}^{2}-\sigma_{1}^{2}} \ln \left[-\frac{\alpha_{1}\left(2 W_{s}^{2}+\sigma_{2}^{2}\right)^{3 / 2}}{\alpha_{2}\left(2 W_{s}^{2}+\sigma_{1}^{2}\right)^{3 / 2}}\right]}$.

Here we remember that $\alpha_{1} /\left(2 W_{s}^{2}+\sigma_{1}^{2}\right)^{3 / 2}>\left|\alpha_{2}\right| /\left(2 W_{s}^{2}+\right.$ $\left.\sigma_{2}^{2}\right)^{3 / 2}$ [see Eq. (9)]. Equation (30) reveals that if $\sigma_{1}>\sigma_{2}$, then the potential is always attractive (no real solution exists), independent of the spatial separation of the solitons, whereas if $\sigma_{2}>\sigma_{1}$, it will always have a transition point $\xi_{t}$ such that for $\xi<\xi_{t}$, it is attractive, while for $\xi>\xi_{t}$, it is repulsive.

These properties of the potential are illustrated in Fig. 14(b) for $\alpha_{1}>\left|\alpha_{2}\right|$. Here the blue color indicates an always attractive potential, while the red color indicates that the potential is either attractive or repulsive depending on the separation of the solitons. We do not depict the potential properties for $\left|\alpha_{2}\right|>$ $\alpha_{1}$, since in this regime one must have $\sigma_{2}>\sigma_{1}$ for a stationary solution to exist, and the figure would be trivial.

A simple analysis of Eq. (30) shows that $\xi_{t}$ decreases with increasing power due to the decrease in $W_{s}$ with increasing power. This is because the higher power of the solitons leads to lower (normalized) intensity in the overlap region, and thus a lower (normalized) refractive index in the center between the solitons (when $\alpha_{1}>\left|\alpha_{2}\right|$ ). The dependence on the width of the nonlinearities is similar to that depicted in Fig. 9.

The physical reason for soliton attraction and repulsion is the same nonlinear refractive index change as discussed in the case of coherent soliton interaction. For completeness, we illustrate in Fig. 15 the light intensity distribution and corresponding nonlinear refractive index $\Delta n(x)$ for two close and well-separated solitons. Again, in the first instance, the index between solitons increases, giving rise to their attraction. For well-separated solitons, $\Delta n(x)$ decreases in the region between solitons, leading to their repulsion.

It is important to stress that in media with only a selffocusing nonlocal nonlinearity, mutually incoherent solitons will always attract each other according to the potential in Eq. (30). This is in agreement with the literature [2,5]. Thus, the fact that mutually incoherent solitons may repel for sufficiently large spatial separations is a pure consequence of the 

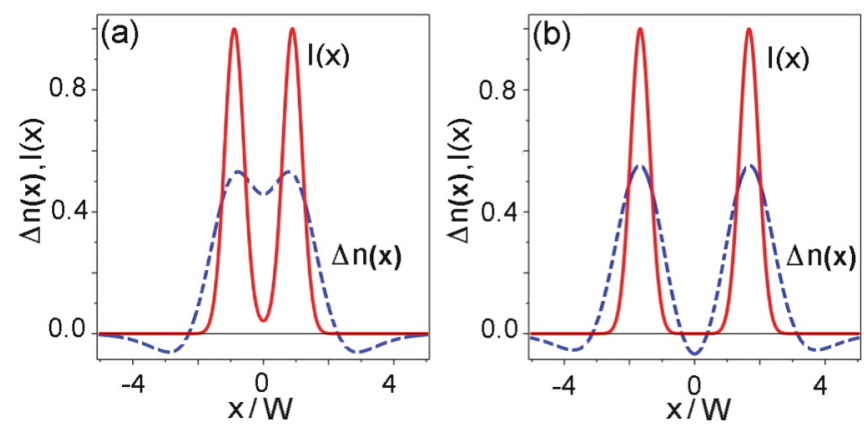

FIG. 15. (Color online) Intensity (solid red line) and nonlinear refractive index change (dashed blue line) of two incoherent solitons with spatial separation of (a) $0.8 \xi_{t}$ and (b) $1.5 \xi_{t}$, where $\xi_{t} \approx 0.44$. The parameters are $P=50, \alpha_{1}=2, \alpha_{2}=-1, \sigma_{1}=1$, and $\sigma_{2}=2$.

competing self-defocusing nonlinearity. Repulsion between mutually incoherent solitons has been observed experimentally in photorefractive media some time ago [64]. However, in that case, the behavior originated from a two-dimensional charge distribution in the process of the formation of screening photorefractive solitons.

\section{NUMERICAL SIMULATIONS OF INCOHERENT SOLITON INTERACTION}

In this section, we will numerically test the validity of the variational results obtained above.

To this end, for given sets of medium parameters $\alpha_{j}, \sigma_{j}$ ( $j=1,2)$ and power $P$, Eq. (9) is solved and the stationary width $W_{s}$ and amplitude $A_{s}$ are found. When these parameters are known, the transition separation $\xi_{t}$, if it exists, can be determined from Eq. (30). The functions given by Eqs. (24) and (25) are used as initial conditions in Eqs. (19) and (20), which are numerically propagated using a split-step Fourier scheme.

We showed previously that for $\sigma_{2}>\sigma_{2}$, mutually incoherent solitons will either attract or repel, depending on their spatial separation. This holds independently of the relative size of $\alpha_{1}$ and $\alpha_{2}$.

Figure 16(a) shows the propagation of two mutually incoherent solitons with initial separation $\xi=\xi_{t}$. The depicted intensity is the sum of the individual intensities, $I=|u|^{2}+$ $|v|^{2}$, normalized to the maximum input intensity, as it is in all of the figures below. The power of the individual solitons is $P=50$ and the medium parameters are $\alpha_{1}=2, \alpha_{2}=-1$,
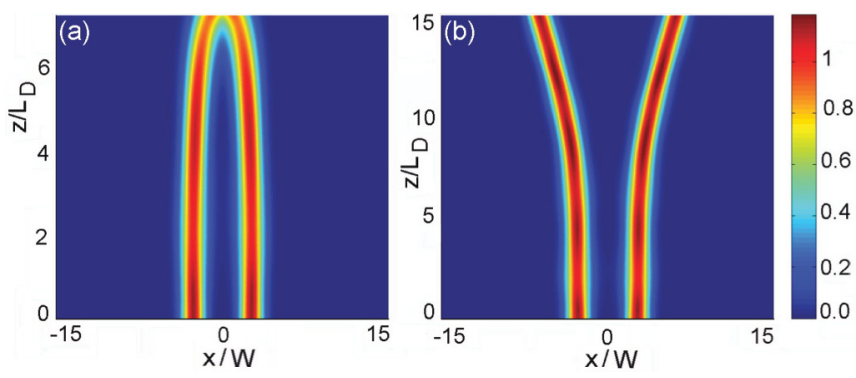

FIG. 16. (Color online) Trajectories of incoherently interacting solitons for $\alpha_{1}>\left|\alpha_{2}\right|$ with power $P=50$ and initial separation (a) $\xi=\xi_{t}$ and (b) $\xi=1.02 \xi_{t}$, where $\xi_{t} \approx 1.09$. The medium parameters are $\alpha_{1}=2, \alpha_{2}=-1, \sigma_{1}=1$, and $\sigma_{2}=2$.
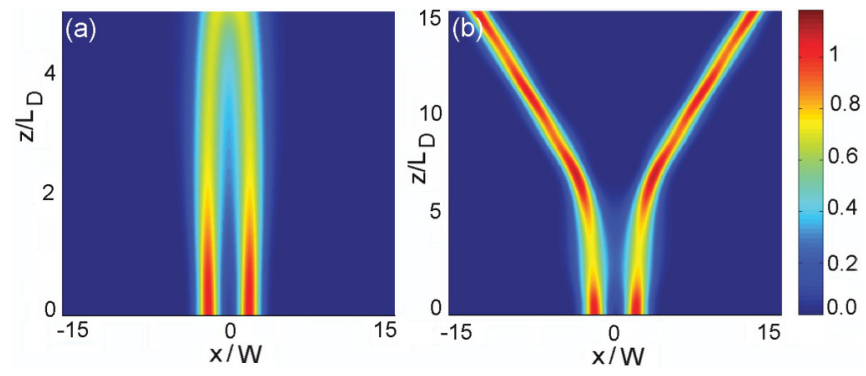

FIG. 17. (Color online) Trajectories of incoherently interacting solitons for $\alpha_{1}<\left|\alpha_{2}\right|$ with power $P=50$ and initial separation (a) $\xi=\xi_{t}$ and (b) $\xi=1.02 \xi_{t}$, where $\xi_{t} \approx 0.97$. The medium parameters are $\alpha_{1}=1, \alpha_{2}=-2, \sigma_{1}=1$, and $\sigma_{2}=3$.

$\sigma_{1}=1$, and $\sigma_{2}=2$. The initial transverse velocity $B$ of the solitons is zero. We observe attraction between the two solitons and, eventually, collision. If the initial separation between them is increased to $\xi=1.02 \xi_{t}$, as shown in Fig. 16(b), then the solitons repel each other, as predicted by the variational calculations. The solitons will continue to move apart from each other. The initial separation of the solitons has to be increased to $\sim 1.02 \xi_{t}$ in order to observe repulsion due to the inaccuracy of the approximate variational results.

Figures 17(a) and 17(b) show the same scenario for incoherently interacting solitons in media with $\left|\alpha_{2}\right|>\alpha_{1}$. In the simulation shown in the first of these figures, the initial separation is $\xi=\xi_{t}$, and we observe that the solitons attract each other. When the initial separation is increased to $\xi=1.02 \xi_{t}$, the solitons repel, as shown in the latter figure. The power of the individual solitons is $P=50$ and the medium parameters are $\alpha_{1}=1, \alpha_{2}=-2, \sigma_{1}=1$, and $\sigma_{2}=3$.

If the interacting solitons in Fig. 16(a) are propagated longer, then they collide, as depicted in Fig. 18(a). Due to the nonintegrability of the governing propagation equations (19) and (20), the collision is inelastic, and thus, the number of solitons may not be conserved in collision events. Figure 18(b) shows the input and output intensity profiles.

Additional numerical simulations (not shown) reveal that in media with $\sigma_{1}>\sigma_{2}$, mutually incoherent solitons are always attracting each other, independently of their spatial separation, as predicted by the variational calculations.
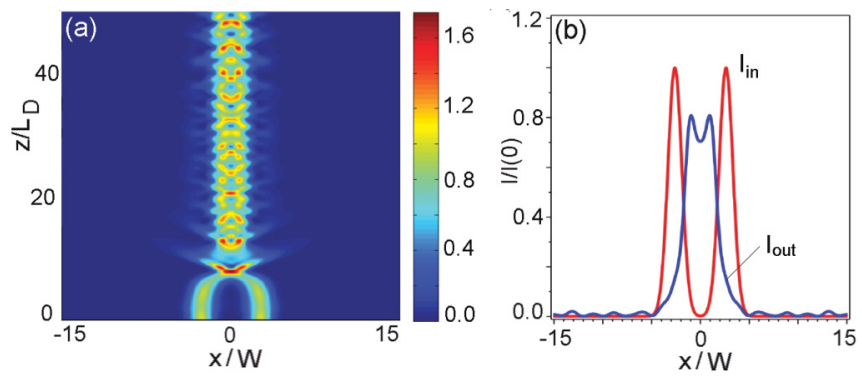

FIG. 18. (Color online) Extended trajectories of incoherently colliding solitons from Fig. 16. The power $P=50$ and initial separation $\xi=\xi_{t}$, where $\xi_{t} \approx 1.09$. The medium parameters are $\alpha_{1}=2, \quad \alpha_{2}=-1, \sigma_{1}=1$, and $\sigma_{2}=2$. (b) Normalized input $\left(z / L_{D}=0\right)$ (red line) and output $\left(z / L_{D}=50\right)$ (blue line) intensity profiles. 

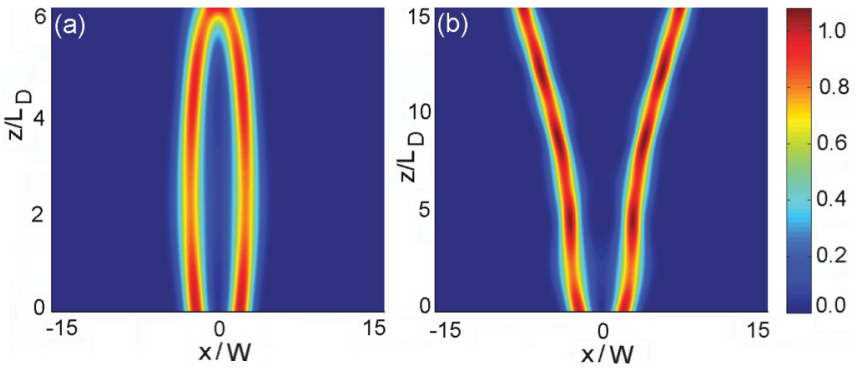

FIG. 19. (Color online) Trajectories of colliding mutually incoherent solitons with power $P=50$ and initial separation $\xi=0.75 \xi_{t}$, where $\xi_{t} \approx 1.09$. Initial velocity (a) $B=1.10$ and (b) $B=1.21$. The medium parameters are $\alpha_{1}=2, \alpha_{2}=-1, \sigma_{1}=1$, and $\sigma_{2}=2$.

So far we have only considered the effective particle equation, given by Eq. (28), in the case of solitons with zero initial relative transverse velocity, i.e., $B=0$. In this case, the initial energy of the "effective" particle is simply given by its potential energy, i.e., $E=V(\xi)$. If $\xi<\xi_{t}$, then we have observed attraction between the solitons, and if $\xi>\xi_{t}$, then we have observed repulsion. However, in the same way that a classical particle may be able to overcome a potential barrier if it has an initial kinetic energy, the outcome of the incoherent soliton interaction may change if the solitons have an initial velocity $B \neq 0$.

We consider the potential in Fig. 14(a) for $\sigma_{2}>\sigma_{1}$. If the spatial separation of the solitons is, say, $0.75 \xi_{t}$, then the shape of the potential tells that the solitons will attract. But if the solitons are given an initial velocity (the effective particle is given an initial kinetic energy) $B$ such that $(2 B)^{2}>V\left(\xi_{t}\right)-$ $V\left(0.75 \xi_{t}\right) \Rightarrow B>1.10$, then we would expect the solitons to "escape" the attractive force and move away from each other.

Figure 19(a) shows the propagation of two mutually incoherent solitons with initial separation $\xi=\xi_{t}$ and initial velocity $B=1.10$. We observe that even though the solitons have an initial outgoing velocity, they are captured by the attractive force between them and eventually collide. If the initial velocity is increased by $10 \%$, then the solitons get a sufficiently high kinetic energy to escape from the attractive potential, and they move away from each other, as depicted in Fig. 19(b).

Similarly, if the spatial separation of the solitons is larger than $\xi_{t}$, say, $\xi=1.25 \xi_{t}$, then the shape of the potential in Fig. 14(a) indicates that the solitons will repel. But if they are given an initial velocity $B$ such that $(2 B)^{2}>V\left(\xi_{t}\right)-$ $V\left(1.25 \xi_{t}\right) \Rightarrow B<-0.66$, then we would expect them to overcome the repulsive force and collide.
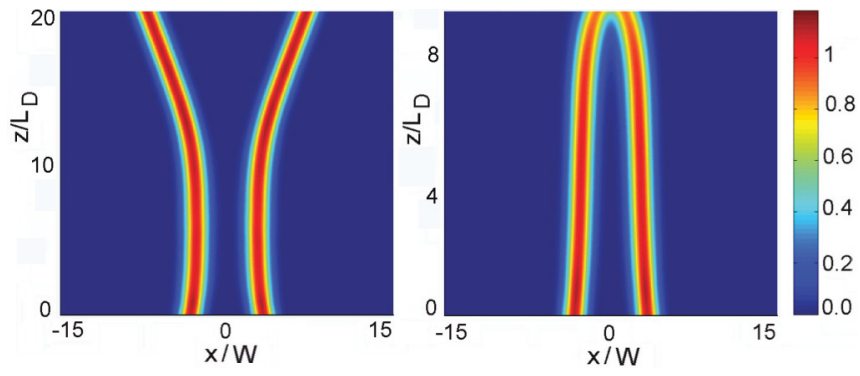

FIG. 20. (Color online) Trajectories of interacting mutually incoherent solitons with power $P=50$ and initial separation $\xi=1.25 \xi_{t}$, where $\xi_{t} \approx 1.09$. Initial velocity (a) $B=-0.63$ and (b) $B=-0.66$. The medium parameters are $\alpha_{1}=2, \alpha_{2}=-1, \sigma_{1}=1$, and $\sigma_{2}=2$.

Figures 20(a) and 20(b) show the propagation of two mutually incoherent solitons with initial separation $\xi=\xi_{t}$ and initial velocity $B=-0.63$ and $B=-0.66$, respectively. We observe that when the initial ingoing velocity is too low (numerically), the repulsive force between the solitons is too strong to allow the solitons to collide. But when the velocity is increased, the solitons overcome the repulsive potential and collide [Fig. 20(b)].

\section{CONCLUSION}

In conclusion, in this work we studied the formation and interaction of solitons in nonlocal media with competing nonlinearities. We used a variational approach to find stationary soliton solutions and found them to be stable. We then employed a variational technique to investigate the interaction between identical solitons. We found that solitons may repel or attract depending on their separation. In particular, while nonlocality induces attraction of nearby solitons, the competition between self-focusing and defocusing may induce repulsion of well-separated solitons. We investigated the dynamics of soliton propagation and interaction using numerical simulations. Our numerical simulations appear to be in perfect agreement with the analytical results obtained with the variational method.

\section{ACKNOWLEDGMENTS}

This work was supported by the Australian Research Council. M.B. acknowledges support from the Danish Council for Independent Research (Femto-VINIR, Project No. 274-08-0479).
[1] Optical Solitons - Theory and Experiment, edited by J. R. Taylor (Cambridge University Press, Cambridge, UK, 1992).

[2] Yu. S. Kivshar and G. Agrawal, Optical Solitons: From Fibers to Photonic Crystals (Academic, San Diego, 2003).

[3] K. E. Strecker, G. B. Patridge, A. G. Truscott, and R. G. Hulet, Nature (London) 417, 150 (2002).

[4] L. Khaykovich, F. Scherck, G. Ferrari, T. Bourdel, J. Cubizolles, L. D. Carr, Y. Castin, and C. Salomon, Science 296, 1290 (2002).
[5] G. I. Stegeman and M. Segev, Science 286, 1518 (1999).

[6] V. E. Zakarov and A. B. Shabat, Sov. Phys. JETP 37, 823 (1973).

[7] S. Cowan, R. H. Enns, S. S. Rangnekar, and S. Sanghera, Can. J. Phys. 64, 311 (1986); S. Gatz and J. Herrmann, Opt. Lett. 17, 484 (1992); W. Krolikowski and S. A. Holmstrom, ibid. 22, 369 (1997).

[8] W. Krolikowski, O. Bang, N. I. Nikolov, D. Neshev, J. Wyller, J. J. Rasmussen, and D. Edmundson, J. Opt. B 6, S288 (2004). 
[9] A. Snyder and J. Mitchell, Science 276, 1538 (1997).

[10] F. W. Dabby and J. B. Whinnery, Appl. Phys. Lett. 13, 284 (1968).

[11] S. Skupin, M. Saffman, and W. Krolikowski, Phys. Rev. Lett. 98, 263902 (2007).

[12] D. Suter and T. Blasberg, Phys. Rev. A 48, 4583 (1993).

[13] E. A. Ultanir, D. Michaelis, F. Lederer, and G. I. Stegeman, Opt. Lett. 28, 251 (2003).

[14] S. Burger, K. Bongs, S. Dettmer, W. Ertmer, K. Sengstock, A. Sanpera, G. V. Shlyapnikov, and M. Lewenstein, Phys. Rev. Lett. 83, 5198 (1999).

[15] F. Dalfovo, S. Giorgini, L. P. Pitaevski, and S. Stringari, Rev. Mod. Phys. 71, 463 (1999).

[16] T. Lahaye, C. Menotti, L. Santos, M. Lewenstein, and T. Pfau, Rep. Prog. Phys. 72, 126401 (2009).

[17] E. Braun, L. P. Faucheux, and A. Libchaber, Phys. Rev. A 48, 611 (1993).

[18] G. Assanto and M. Peccianti, IEEE J. Quantum Electron. 39, 13 (2003).

[19] C. Conti, M. Peccianti, and G. Assanto, Phys. Rev. Lett. 91, 073901 (2003).

[20] N. I. Nikolov, D. Neshev, O. Bang, and W. Z. Krolikowski, Phys. Rev. E 68, 036614 (2003).

[21] J. Wyller, W. Krolikowski, O. Bang, D. E. Petersen, and J. J. Rasmussen, Physica D 227, 8 (2007).

[22] F. O. Ilday, K. Beckwitt, Y.-F. Chen, H. Lim, and F. W. Wise, J. Opt. Soc. Am. B 21, 376 (2004).

[23] M. Bache, O. Bang, J. Moses, and F. W. Wise, Opt. Lett. 32, 2490 (2007).

[24] M. Bache, O. Bang, W. Krolikowski, J. Moses, and F. W. Wise, Opt. Express 16, 3273 (2008).

[25] S. K. Turitsyn, Teor. Mat. Fiz. 64, 226 (1985).

[26] O. Bang, W. Krolikowski, J. Wyller, and J. J. Rasmussen, Phys. Rev. E 66, 046619 (2002).

[27] D. Briedis, D. E. Petersen, D. Edmundson, W. Krolikowski, and O. Bang, Opt. Express 13, 435 (2005).

[28] A. I. Yakimenko, Y. A. Zaliznyak, and Y. Kivshar, Phys. Rev. E 71, 065603 (2005).

[29] Y. Y. Lin, R. K. Lee, and Y. S. Kivshar, J. Opt. Soc. Am. B 25, 576 (2008).

[30] A. Armaroli, S. Trillo, and A. Fratalocchi, Phys. Rev. A 80, 053803 (2009).

[31] S. Skupin, O. Bang, D. Edmundson, and W. Krolikowski, Phys. Rev. E 73, 066603 (2006).

[32] M. Peccianti, K. A. Brzdakiewicz, and G. Assanto, Opt. Lett. 27, 1460 (2002).

[33] M. Peccianti, C. Conti, G. Assanto, A. De Luca, and C. Umeton, Appl. Phys. Lett. 81, 3335 (2002).

[34] A. Fratalocchi, M. Peccianti, C. Conti, and G. Assanto, Mol. Cryst. Liq. Cryst. 421, 197 (2004).

[35] N. Nikolov, D. Neshev, W. Krolikowski, O. Bang, J. J. Rasmussen, and P. L. Christiansen, Opt. Lett. 29, 286 (2004).

[36] P. D. Rasmussen, O. Bang, and W. Krolikowski, Phys. Rev. E 72, 066611 (2005).
[37] A. Dreischuh, D. N. Neshev, D. E. Petersen, O. Bang, and W. Krolikowski, Phys. Rev. Lett. 96, 043901 (2006).

[38] Q. Kong, Q. Wang, O. Bang, and W. Krolikowski, Phys. Rev. A 82, 013826 (2010).

[39] J. Mitchell and A. W. Snyder, J. Opt. Soc. Am. B 16, 236 (1999).

[40] F. W. Ye, Y. V. Kartashov, and L. Torner, Phys. Rev. A 77, 043821 (2008).

[41] X. Hutsebaut, C. Cambournac, M. Haelterman, A. Adamski, and K. Neyts, Opt. Commun. 233, 211 (2004).

[42] C. Rotschild, M. Segev, Z. Xu, Y. V. Kartashov, and L. Torner, Opt. Lett. 31, 3312 (2006)

[43] M. L. Quiroga-Teixeiro and H. Michinel, J. Opt. Soc. Am. B 14, 2004 (1997); M. L. Quiroga-Teixeiro, A. Berntson, and H. Michinel, ibid. 16, 1697 (1999); I. Towers, A. V. Buryak, R. A. Sammut, B. A. Malomed, L. C. Crasovan, and D. Mihalache, Phys. Lett. A 288, 292 (2001); B. A. Malomed, L.-C. Crasovan, and D. Mihalache, Physica D 161, 187 (2002).

[44] J. F. Corney and O. Bang, Phys. Rev. E 64, 047601 (2001).

[45] J. Cuevas, B. A. Malomed, P. G. Kevrekidis, and D. J. Frantzeskakis, Phys. Rev. A 79, 053608 (2009).

[46] A. Griesmaier, J. Stuhler, T. Koch, M. Fattori, T. Pfau, and S. Giovanazzi, Phys. Rev. Lett. 97, 250402 (2006).

[47] S. Sinha and L. Santos, Phys. Rev. Lett. 99, 140406 (2007).

[48] L. Bergé, O. Bang, J. J. Rasmussen, and V. K. Mezentsev, Phys. Rev. E 55, 3555 (1997).

[49] O. Bang, Y. S. Kivshar, and A. V. Buryak, Opt. Lett. 22, 1680 (1997).

[50] X. Liu, L. Qian, and F. W. Wise, Opt. Lett. 24, 1777 (1999).

[51] S. Ashihara, J. Nishina, T. Shimura, and K. Kuroda, J. Opt. Soc. Am. B 19, 2505 (2002).

[52] M. Warenghem, J. F. Blach, and J. F. Henninot, J. Opt. Soc. Am. B 25, 1882 (2008).

[53] I. B. Burgess, M. Peccianti, G. Assanto, and R. Morandotti, Phys. Rev. Lett. 102, 203903 (2009).

[54] D. Mihalache, D. Mazilu, F. Lederer, L. C. Crasovan, Y. V. Kartashov, L. Torner, and B. A. Malomed, Phys. Rev. E 74, 066614 (2006).

[55] Y. V. Kartashov, V. A. Vysloukh, and L. Torner, Phys. Rev. A 79, 013803 (2009).

[56] Z. Xu, Y. V. Kartashov, and L. Torner, Phys. Rev. E 73, 055601 (2006).

[57] Y. Du, Z. Zhou, H. Tian, and D. Liu, J. Opt. 13, 015201 (2010).

[58] Z. Zhou, Y. Du, C. Hou, H. Tian, and Y. Wang, J. Opt. Soc. Am. B 28, 1583 (2011).

[59] D. Anderson, Phys. Rev. A 27, 3135 (1983); A. Anderson, M. Lisak, and A. Berntson, Pramana: J. Phys. 57, 917 (2001).

[60] B. Malomed, Prog. Opt. 43, 71 (2002).

[61] N. G. Vakhitov and A. A. Kolokolov, Radiophys. Quantum Electron. 16, 783 (1973).

[62] Z. Xu, Y. V. Kartashov, and L. Torner, Opt. Lett. 23, 3171 (2005).

[63] D. Anderson and M. Lisak, Phys. Scr. 33, 193 (1986).

[64] W. Krolikowski, M. Saffman, B. Luther-Davies, and C. Denz, Phys. Rev. Lett. 80, 3240 (1998). 